
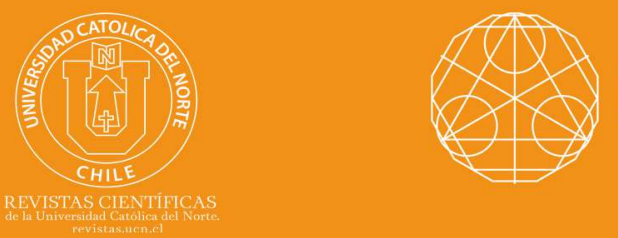

\title{
On ideal sumset labelled graphs
}

\author{
Jincy P Mathai ${ }^{1}$ \\ Sudev Naduvath ${ }^{2}$ @ orcid.org/0000-0001-9692-4053 \\ Satheesh Sreedharan ${ }^{3}$ (i) orcid.org/0000-0001-9703-6154
}

${ }^{1}$ Muthoot Institute of Technology \& Science, Dept. of Mathematics, Puthencruz, KL, India. pjincyparappattu@gmail.com

${ }^{2}$ CHRIST (Deemed to be University), Dept. of Mathematics, Bangalore, KA, India.

sudev.nk@christuniversity.in

${ }^{3}$ Vidya Academy of Science \& Technology, Dept. of Mathematics, Thrissur, KL, India.

satheesh.s@vidyaacademy.ac.in

Received: February 2020 | Accepted: October 2020

\section{Abstract:}

The sumset of two sets $A$ and $B$ of integers, denoted by $A+B$, is defined as $A+B$ $=\{a+b: a \in A, b \in B\}$. Let $X$ be a non-empty set of non-negative integers. $A$ sumset labelling of a graph $G$ is an injective function $f: V(G) \rightarrow P(X)-\{\varnothing\}$ such that the induced function $f^{*}: E(G) \rightarrow P(X)-\{\varnothing\}$ is defined by $f^{+}(u v)=f(u)+f(v)$ $\forall u v \in E(G)$. In this paper, we introduce the notion of ideal sumset labelling of graph and discuss the admissibility of this labelling by certain graph classes and discuss some structural characterization of those graphs.

Keywords: Graph labelling; Set-labelling; Sumset labelling; Ideal sumset labelling.

MSC (2020): 05C78, 11B13.

\section{Cite this article as (IEEE citation style):}

J. P. Mathai, S. Naduvath, and S. Sreedharan, "On ideal sumset labelled graphs", Proyecciones (Antofagasta, On line), vol. 40, no. 2, pp. 371-384, 2021, doi: 10.22199/issn.0717-62792021-02-0022

Article copyright: (C) 2021 Jincy P. Mathai, Sudev Naduvath, and Satheesh Sreedharan. This is an open access article distributed under the terms of the Creative Commons License, which permits unrestricted use and distribution provided the original author and source are credited. 


\section{Introduction}

For all terms and definitions in graph theory, we refer to $[2,7,16]$. For further terminology of graph classes, see $[3,5]$. Unless mentioned otherwise, all graphs we consider in this paper are simple, finite and connected.

Graph labelling has emerged as a potential research area in graph theory soon after the introduction of $\beta$-valuations of graphs in [13]. Later, analogous to number valuations of graphs, the notion of set-valuations has been introduced in [1] as follows: A set-labelling of a graph $G$ can generally be considered as an assignment of the vertices of a graph to the subsets of a non-empty set $X$ in an injective manner such that the set-labels of the edges of $G$ are obtained by taking the symmetric difference of the set-labels of their end vertices.

The sumset of two sets $A$ and $B$ of integers, denoted by $A+B$, is defined as $A+B=\{a+b: a \in A, b \in B\}$ (see [12]). Note that the sumset can be defined only for number sets.

Using the concepts of sumsets of number sets, the notion of sumset labelling of graphs have been introduced in [8] as follows: Let $X$ be a non-empty set of non-negative integers. A sumset labelling (or an integer additive set-labelling) is an injective function $f: V(G) \rightarrow \mathcal{P}(X)-\{\emptyset\}$ such that the induced function $f^{+}: E(G) \rightarrow \mathcal{P}_{0}(X)=\mathcal{P}(X)-\{\emptyset\}$ is defined by $f^{+}(u v)=f(u)+f(v) \forall u v \in E(G)$ (see [8]). A graph $G$ which admits a sumset labelling is called a sumset valued graph (or an integer additive set-Labelled graph). It is proved in [6] that for a sumset labelled graph $G$, $|f(u)|+|f(v)|-1 \leq\left|f^{+}(u v)\right| \leq|f(u)||f(v)|$ for all $u v \in E(G)$.

Initial studies on sumset labelled graphs can be found in $[6,8-11,14,15]$. These papers discussed different types of sumset labelling and sumset labelled graphs. A topological sumset labelling is a sumset labelling $f$ : $V(G) \rightarrow \mathcal{P}_{0}(X)=\mathcal{P}(X)-\{\emptyset\}$ such that $f(V(G)) \cup\{\emptyset\}$ is a topology on $X$ (see [8]). A graceful sumset labelling is a sumset indexer $f: V(G) \rightarrow \mathcal{P}(X)$ such that $f^{+}(E(G))=\mathcal{P}(X)-\{\emptyset,\{0\}\}$ (see [9]).

The above mentioned articles dealt with studies on sumset labelled graphs whose elements receive set-labels from different types of subset collections of $X$. Hence, motivated by these studies, we use some other subset collections to label the elements of a graph $G$ under consideration.

An ideal $\mathcal{J}$ of a non-empty set $X$ (cf. [4]) is a collection of subsets of $X$, which satisfies the following conditions:

(i) $\emptyset \in \mathcal{J}$;

(ii) If $A \in \mathcal{J}$ and $B \subseteq A$, then $B \in \mathcal{J}$; 
(iii) If $A, B \in \mathcal{J}$, then $A \cup B \in \mathcal{J}$.

In this paper, we define a particular type of sumset labelling called the ideal sumset labelling of a graph using the notion of ideals of a set and discuss some structural characteristics of the graphs which admit this new set-labelling.

\section{Ideal Sumset Labelled Graphs}

Using the notion of an ideal of a set, an ideal sumset labelling of a graph $G$ is defined as follows:

Definition 2.1 (Ideal sumset labelling of graphs). Let $X$ be a nonempty set of non-negative integers and $\mathcal{P}(X)$ be its power set. $A$ sumset labelling of a graph $G$ is said to be an ideal sumset labelling if $f(V(G)) \cup\{\emptyset\}$ is an ideal on $X$.

Definition 2.2 (Ideal sumset indexer). An ideal sumset indexer of a graph $G$ is an ideal sumset labelling $f: V(G) \rightarrow \mathcal{P}_{0}(X)$ of $G$ such that the induced function $f^{+}: E(G) \rightarrow \mathcal{P}_{0}(X)$ defined by $f^{+}(u v)=f(u)+$ $f(v) \forall u v \in E(G)$ is also injective.

A graph which admits an ideal sumset labelling is known to be an ideal sumset labelled graph (ISL-graph) and a graph which admits an ideal sumset indexer is known as an ideal sumset indexed graphs. An example to an ISL-graph is provided in Figure 2.1. 


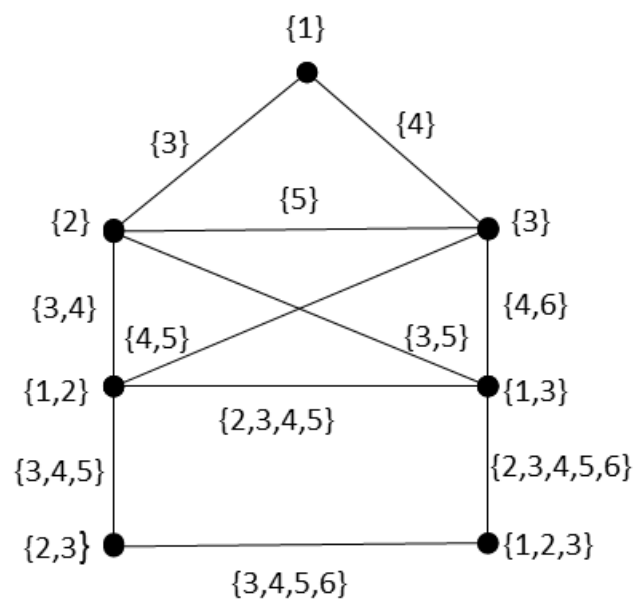

Figure 2.1: An illustration to ISL-graph

Note that all graphs are not ISL-graphs, in general. Hence, the studies on the structural characteristics of ISL-graphs become significant and interesting.

Remark 2.3. If $X \in \mathcal{J}$, then we have $f(V(G))=\mathcal{P}(X)-\{\emptyset\}$. Then, $f$ is also a topological sumset labelling with respect to the discrete topology on the ground set $X$. In other words, $X$ can be the set label of a vertex in $G$ if

(i) $f$ is also a topological sumset labelling;

(ii) $f(V(G)) \cup\{\emptyset\}$ is the discrete topology on $X$.

(iii) $G$ must have $2^{|X|}-1$ vertices.

In view of the above-mentioned facts, hereafter we consider the ideals of $X$, does not contain $X$. The following lemma describes the order of an ISL-graph.

Lemma 2.4. An ISL-graph $G$ has $2^{|A|}-1$ vertices, where $A$ is the maximal element of the corresponding ideal $I=f(V(G)) \cup\{\emptyset\}$. 
Proof. Let $G$ be an ISL-graph with respect to an ideal $I$ of the ground set $X$. Clearly $I=f(V(G)) \cup\{\emptyset\}$. Since $A$ is the maximal element of $I$, all non-empty subsets of $A$ should be the set labels of some vertices of $G$. That is, $f(V(G))=2^{A}-\{\emptyset\}$. Therefore, $|f(V(G))|=|V(G)|=2^{|A|}-1$.

The following theorem describes a necessary and sufficient condition for a sumset labelling $f$ to be an ideal sumset labelling of $G$.

Theorem 2.5. A sumset labelling $f: V(G) \rightarrow \mathcal{P}_{0}(X)$ of a graph $G$ is an ideal sumset labelling of $G$ if and only if $G$ has exactly $2^{|A|}-1$ vertices and $f(V(G)) \cup\{\emptyset\}=\mathcal{P}(A)$ where $A$ is the maximal element of the corresponding ideal $\mathcal{J}=f(V(G)) \cup\{\emptyset\}$.

Proof. Let $f: V(G) \rightarrow \mathcal{P}_{0}(X)$ be an ideal sumset labelling of a graph $G$. Then, necessary part is immediate from Lemma 2.4.

Conversely, let $G$ be a graph with $2^{k}-1$ vertices; $k \in Z^{+}$. Choose $X$, sufficiently large, and let $A$ be a subset of $X$ with cardinality $k$ such that the sumsets of subsets of $A$ must be the subsets of $X$. Note that the collection of all subsets of $A$, will form an ideal of $X$.

Now, label the vertices of $G$, in an injective manner, by non-empty sets in $I$. Clearly, this labelling will be an ideal sumset labelling of $G$. This completes the proof.

Theorem 2.6. Let $G$ be a graph of order $n$, which admits an ideal sumset labelling with respect to a ground set $X$. Then $X$ has at least $\log _{2}|n+1|$ elements.

Proof. Let $G$ be an ISL-graph with $n$ vertices. Then, $n=2^{k}-1$ for some $k$. Hence $k=\log _{2}|n+1|$. Therefore $X$ should have at least $k$ elements to label the graph $G$. Thus, $|X| \geq k$. That is, $|X| \geq \log _{2}|n+1|$.

Definition 2.7 (Uniform Ideal Sumset Labelling). An ideal sumset labelling of a graph $G$ is said to be a uniform ideal sumset labelling (UISL) of $G$ if the cardinality of the set labels of all edges of $G$ are same. If $\left|f^{+}(e)\right|=k \forall e \in E(G)$, then the corresponding ideal sumset labelling is said to be a $k$-uniform ideal sumset labelling.

Figure 2.2 depicts a graph with a uniform ideal sumset labelling. 


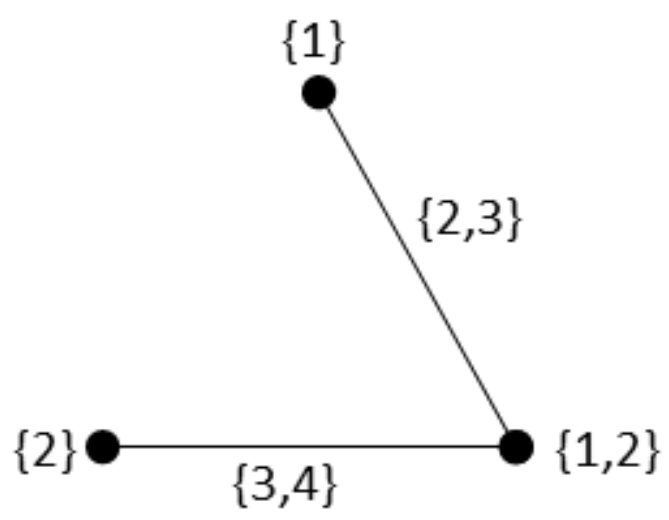

Figure 2.2: An example of a uniform ISL-graph

The notion of a strong ideal sumset labelling of a graph $G$ is introduced as follows:

Definition 2.8 (Strong Ideal Sumset Labelling). An ideal sumset labelling $f: V(G) \rightarrow \mathcal{P}_{0}(X)$ is said to be a strong ideal sumset labelling if $\left|f^{+}(u v)\right|=|f(u)||f(v)|$ for all edges $u v \in E(G)$. 


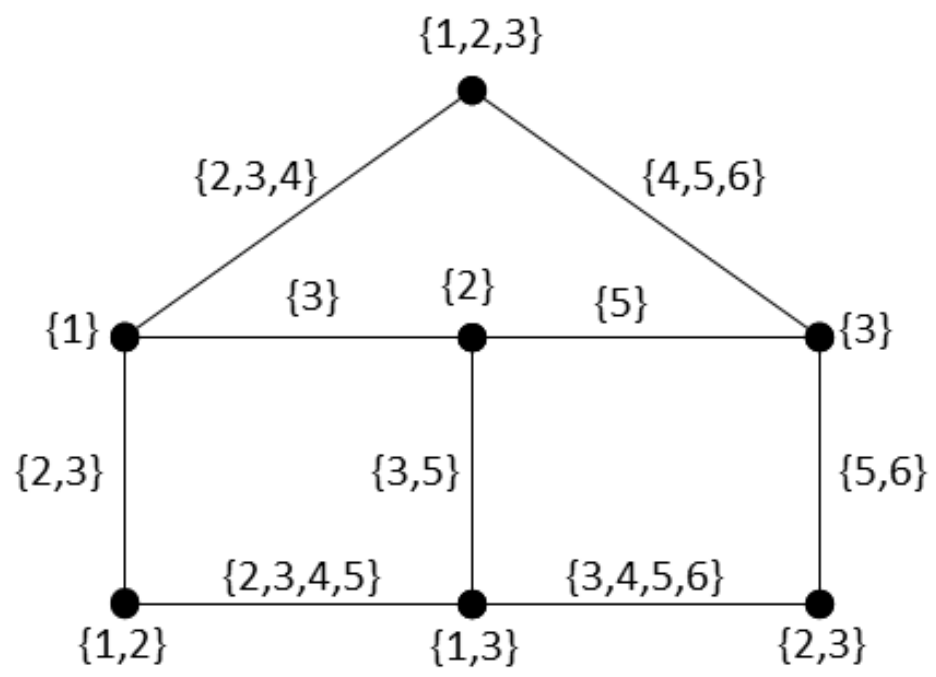

Figure 2.3: An example to strong ISL-graph

Proposition 2.9 provides a necessary condition for an ideal sumset labelling of a graph $G$ to become a strong ideal sumset labelling of $G$.

Proposition 2.9. If a sumset labelled graph $G$ is a strong ISL-graph, then the difference sets of the set labels of any two adjacent vertices in $G$ are disjoint.

Proof. The proof is exactly same as in the case of strong sumset labelling (see $[8]$ ).

The converse of Theorem 2.9 need not be true. This fact can be verified from Figure 2, in which the set-labels of the elements of the graph satisfies the condition $D_{f(u)} \cap D_{f(v)}=\emptyset$ for all $u v \in E(G)$, but it is not a strong ISL-graph. 


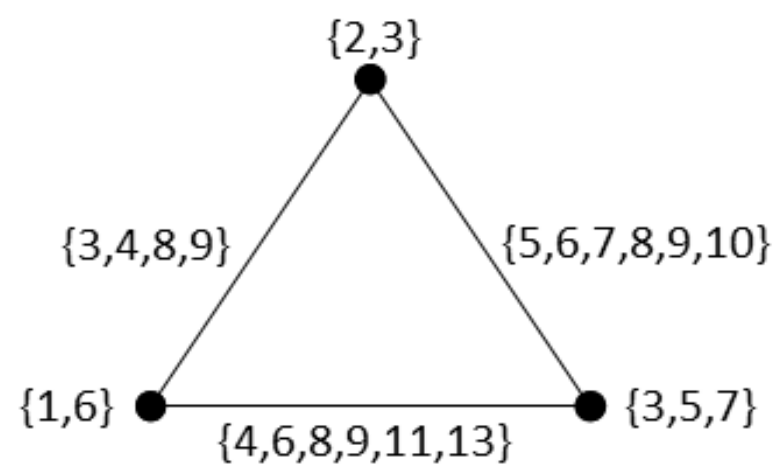

Figure 2.4: A graph which is not a strong ISL-graph

The following theorem describes a necessary and sufficient condition for an ideal sumset labelling of a given graph to become a topological sumset labelling of $G$.

Theorem 2.10. Let $X$ be a non empty finite set of integers. Then, an ideal sumset labelling of a graph $G$ with respect to $X$ is a topological sumset labelling if and only if $0 \in X$ and $f(V(G)) \cup\{\emptyset\}=\mathcal{P}(X)$.

Proof. Let $f$ be an ideal sumset labelling of a graph $G$ with respect to the ground set $X$. Assume that $f$ is a topological sumset labelling of $G$. Then $f(V(G)) \cup\{\emptyset\}$ is a topology on $X$. Therefore, $X \in f(V(G))$. That is, for some vertex $v \in V(G), f(v)=X$. Then, $v$ will be adjacent to a vertex whose set label is $\{0\}$ only. Therefore, $0 \in X$. Since $G$ is an ISL-graph, $f(V(G)) \cup\{\emptyset\}$ is an ideal on $X$ and an ideal is a subset of $\mathcal{P}(X)$. Also, since $X \in f(V(G))$ and $X$ is a subset of itself, $f(V(G)) \cup\{\emptyset\}=\mathcal{P}(X)$. Conversely, assume that $0 \in X$ and $f(V(G)) \cup\{\emptyset\}=\mathcal{P}(X)$. In order to prove that $G$ admits a topological sumset labelling, we have to show that $f(V(G)) \cup\{\emptyset\}$ is a topology on $X$. Since $f(V(G)) \cup\{\emptyset\}=\mathcal{P}(X)$, $X \in f(V(G))$. Also, since $\mathcal{P}(X)$ is the set of all subsets of $X, f(V(G)) \cup$ $\{\emptyset\}$ is closed under arbitrary union and finite intersections. Therefore, $f(V(G)) \cup\{\emptyset\}$ is a topology on $X$. Hence, $G$ admits a topological sumset labelling of $G$.

The following result provides an interesting structural characteristic of a topological ISL-graph. 
Theorem 2.11. If $G$ is an ideal sumset indexed graph which admits topological sumset labelling, then $G$ has exactly $2^{|X|}-2$ pendant vertices which are adjacent to a single vertex of $G$.

Proof. Let $G$ be an ideal sumset indexed graph. Then $G$ has $2^{|X|}-1$ vertices. Since $G$ admits a topological sumset labelling, $0 \in X$ and $f(V(G)) \cup$ $\{\emptyset\}=\mathcal{P}(X)$, by Theorem 2.10. Therefore, there exists a vertex $v$ in $G$ whose set label is $\{0\}$. Now let $u$ be any vertex in $G$ other than $v$. Then, we have to show that $u$ is adjacent to $v$ only. If possible assume that $w$ is a vertex in $G$ and $u$ is adjacent to $w$. Here, we have to consider the following cases:

Case 1: Let $l$ and $k$ be any two nonzero elements in $f(u)$ and let $k$ be the maximal element of $X$. If $l \in f(w)$, then $l+k \in f^{+}(u w)$ and is greater than $k$ which is a contradiction to the fact that $f^{+}(u w)=f(u)+f(w) \subseteq X$, as $f$ is a sumset labelling of $G$.

Case 2: If $f(u)$ and $f(w)$ does not contain maximal element, then the edge labelling will not be injective. Therefore, $u$ is adjacent to a unique vertex that has set label $\{0\}$. Hence $u$ is a pendant vertex of $G$. Since there are $2^{|X|}-1$ vertices in $G, u$ is any one of these vertices other than the vertex having set label $\{0\}$ and the vertex having set label $\{0\}$ is not a pendant vertex. Therefore, there are $2^{|X|}-2$ pendant vertices in an ideal sumset indexed graph which admits topological sumset labelling.

Lemma 2.12. Let $X$ be a nonempty set of non negative integers. If an ideal sumset labelling $f: V(G) \rightarrow \mathcal{P}_{0}(X)$ is a graceful sumset labelling of a given graph $G$, then $\{0\}$ must be the set label of a vertex in $G$.

Proof. Let $f: V(G) \rightarrow \mathcal{P}_{0}(X)$ be an ideal sumset indexer. Assume that $f$ is a graceful sumset labelling. Then $f^{+}(E(G))=\mathcal{P}(X)-\{\emptyset,\{0\}\}$. We have to prove that $\{0\}$ is a set label of a vertex in $G$. Suppose $\{0\}$ not be the set label of any vertex in $G$. Then there exists at least one edge whose set-label is not a subset of $X$ since, $X$ is a non empty set of non negative integers. This is a contradiction to the assumption that $f$ is a graceful sumset labelling. Therefore, $\{0\}$ must be the set-label of a vertex in $G$.

In view of the above fact, a necessary and sufficient condition for an ideal sumset labelling of a graph $G$ to become a graceful sumset labelling is explained in the following result. 
Theorem 2.13. An ideal sumset labelled graph $G$ is a graceful sumset Labelled graph if and only if

(i) $\{0\} \in f(V(G))$;

(ii) $f(V(G)) \cup\{\emptyset\}=\mathcal{P}(X)$;

(iii) $G$ has exactly $2^{|X|}-2$ pendant vertices which are adjacent to $\{0\}$.

Proof. Let $G$ be an ideal sumset labelled graph. Then $G$ has $2^{|X|}-1$ vertices by Lemma 2.4. Suppose that $G$ is a graceful sumset Labelled graph. Then $f^{+}(E(G))=\mathcal{P}(X)-\{\emptyset,\{0\}\}$. By Lemma 2.12, $\{0\}$ must be the set-label of a vertex in $G$. That is, $\{0\} \in f(V(G))$. Since $G$ is an ideal sumset indexed graph, $f(V(G)) \cup\{\emptyset\}$ is an ideal on $X$. Therefore, we have $f(V(G)) \cup\{\emptyset\} \subseteq \mathcal{P}(X)$. Now since $f^{+}(E(G))=\mathcal{P}(X)-\{\emptyset,\{0\}\}$, $X \in f^{+}(E(G))$. Therefore, $X \in f(V(G))$ also, otherwise the sumset of the set-label of $X$ and the set-label of any vertex, other than $\{0\}$ will not be a subset of $X$. Thus, $f(V(G)) \cup\{\emptyset\}=\mathcal{P}(X)$. Next, we have to prove that $G$ has exactly $2^{|X|}-2$ pendant vertices which are adjacent to $\{0\}$. Since $G$ has $2^{|X|}-1$ vertices, there are $2^{|X|}-2$ vertices other than $\{0\}$. All these $2^{|X|}-2$

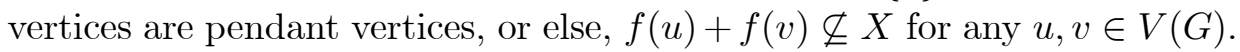
Conversely, assume that $G$ is an ideal sumset indexed graph which satisfies (i), (ii), (iii). To prove that $G$ is a graceful sumset labelled graph. In a sumset labelled graph, we consider only non empty sets for labelling the graph. Therefore, $\emptyset \notin f(V(G))$ and hence $\emptyset \notin f^{+}(E(G))$. Also $\{0\}$ cannot be the set-label of any edge of $G$. Therefore, $f^{+}(E(G))=P(X)-\{\emptyset,\{0\}\}$ by (ii). Thus, $G$ is a graceful sumset labelled graph.

\section{Ideal Sumset Labelling of Some Fundamental Graph Classes}

The following result describes a necessary and sufficient condition for a complete graph to admit an ideal sumset labelling.

Theorem 3.1. A complete graph $K_{n}$ admits ideal sumset labelling if and only if $n=2^{k}-1$, for any integer $k \geq 1$.

Proof. Assume that $K_{n}$ admits ideal sumset labelling. Then, by Theorem $2.5, K_{n}$ has exactly $2^{k}-1$ vertices where $k$ is the cardinality of the maximal element of the corresponding ideal $\mathcal{J}=f(V(G)) \cup\{\emptyset\}$. Therefore, $n=2^{k}-1$. Conversely, assume that $n=2^{k}-1$ for any integer $k \geq 1$. Then, 
$n+1=2^{k}$ and hence $f(V(G)) \cup\{\emptyset\}=\mathcal{P}(X)$. Therefore, by Theorem 2.5, $K_{n}$ admits ideal sumset labelling.

Theorem 3.2. A star graph $K_{1, m}$ admits ideal sumset labelling if and only if $m=2^{n}-2$ for any integer $n>1$.

Proof. Let $v$ be the vertex of degree $d(v)>1$ and $\left\{v_{1}, v_{2}, \ldots, v_{m}\right\}$ be the vertices in $K_{1, m}$ which are adjacent to $v$. Let $X$ be a set of positive integers. First, assume that $G=K_{1, m}$ admits ideal sumset labelling. Then, by Theorem 2.5, $f(V(G)) \cup\{\emptyset\}=\mathcal{P}(X)$. Hence, $|f(V(G)) \cup\{\emptyset\}|=2^{|X|}$. Hence, $\mid f\left(V(G) \mid=2^{|X|}-1\right.$. Thus, $1+m=2^{n}-1$. Therefore $m=2^{n}-2$. Conversely, consider $G=K_{1, m}$ where $m=2^{n}-2$ for any integer $n>1$. Choose a set $X$ with cardinality $n$. Define a set labelling of $G$ which assigns any of the singleton set to the central vertex of $G$ and the other non empty subsets of $X$ to the pendant vertices of $G$. Clearly, this labelling is a sumset labelling of $G$. Also, $f(V(G)) \cup\{\emptyset\}=\mathcal{P}(X)$. Therefore, $f$ is an ideal sumset labelling of $G$.

\section{Graphical Realisation of Ideals}

We have so far discussed the labelling of given graphs. Given an ideal $\mathcal{J}$ of a set $X$, it is also interesting to check whether we can construct a graph $G$ such that $G$ admits an ideal sumset labelling with respect to $\mathcal{J}$. This leads us to the following notion.

Definition 4.1 ( $\mathcal{J}$ - Realisation of Ideals). Let $f$ be an ideal sumset labelling of a graph $G$ with respect to the ground set $X$. Then, $\mathcal{J}=$ $f(V(G)) \cup\{\emptyset\}$ is an ideal on $X$. Hence the graph $G$ is said to be an $\mathcal{J}$-graphical realisation (or simply $\mathcal{J}$-realisation) of $\mathcal{J}$.

The following theorem establishes the existence of a graphical realisation corresponding to a given ideal of the ground set $X$.

Theorem 4.2. Let $X$ be a non empty finite set of integers. Then, there exists an $\mathcal{J}$-realisation for every ideal $\mathcal{J}$ of $X$.

Proof. Let $\mathcal{J}$ be an ideal of a non empty finite set $X$. We need to construct a graph $G$ whose vertices have set labels taken from $\mathcal{J}$ in an injective manner. Take a star graph $K_{1,2|X|-2}$. Label its central vertex by any singleton set and label the other vertices by remaining $2^{|X|}-2$ non 
empty sets in $\mathcal{J}$. Clearly this labelling is an ideal sumset labelling defined on the star graph $K_{1,2|X|_{-2}}$ and hence $K_{1,2|X|-2}$ is a graphical realisation of $\mathcal{J}$.

Figure 4.1 depicts a $\mathcal{J}$-realisation of an ideal $\mathcal{J}$ of the set $\{1,2,3,4,5\}$.

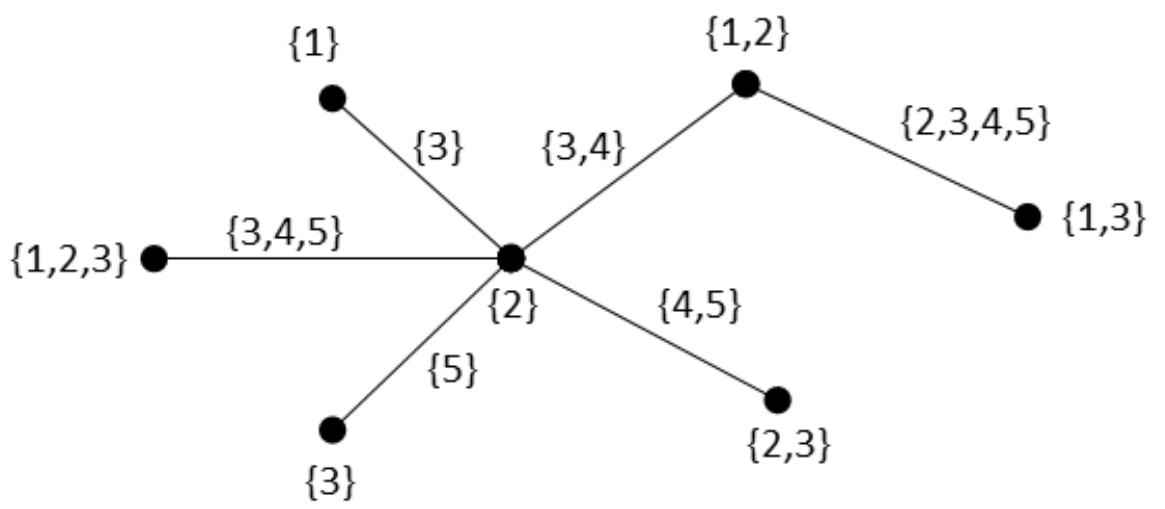

Figure 4.1: Graphical realisation of an ideal of a set $\mathrm{X}$

\section{Conclusion}

In this paper, we have introduced a particular type of sumset labelling of graphs, called the ideal sumset labelling of graphs and discussed the structural characteristics of the graphs which admit this type of labelling. Further intense studies are possible in this direction. It is quite interesting examine the existence of (induced or independent) ideal sumset-labelling for graph operations, graph products and derived graphs of ISL-graphs.

Another promising area in this direction is to explore the relations between ideal sumset labelling and other types of sumset labelling and corresponding characterisations of the graphs which admit these types of labelling. The studies seem to be exciting as it can be extended to the cases when other binary operations of sets are used in place of sumset operation. 


\section{Acknowledgement}

The authors would like to acknowledge the suggestions and critical comments of the anonymous referee which improved the style and content the article significantly. The authors also acknowledge the help rendered by the Centre for Studies in Discrete Mathematics, Thrissur and the Department of Mathematics Vidya Academy of Science \& Technology, Thrissur, India.

\section{References}

[1] B. D. Acharya, "Set valuations of a graph and their applications", MRI lecture notes in applied mathematics, no. 2, 1986.

[2] J. A. Bondy and U. S. R. Murty, Graph theory with applications. London: Macmillan, 1976.

[3] A. Brandstädt, V. B. Le, and J. P. Spinrad, Graph classes: a survey. Philadelphia, PA: SIAM, 1999, doi: 10.1137/ 1.9780898719796

[4] I. Farah, Analytic quotients: theory of liftings for quotients over analytic ideals on the integers. Providence, RI American Mathematical Society, 2000, doi: 10.1090/ memo/ 0702

[5] J. A. Gallian, "A dynamic survey of graph labeling", The electronics journal of combinatorics, vol. DS6, Dec. 2018, doi: 10.37236/ 27

[6] K. A. Germina and N. K. Sudev, "On weakly uniform integer additive set-indexers of graphs", International mathematical forum, vol. 8, no. 37, pp. 1827-1834, 2013, doi: 10.12988/ imf.2013.310188

[7] F. Harary, Graph theory. New Delhi: Narosa, 2001.

[8] S. Naduvath and G Augustine, An introduction of sumset valued graphs. Lambert, Beau Bassin-Rose Hill: Lambert, 2018.

[9] S. Naduvath and K. A. Germina, "A study on integer additive setgraceful of graphs", Southeast Asian bulletin of mathematics, vol. 43, no. 5, pp. 761-772. [On line]. Available: https:/ / bit.ly/ 3bFwyKq 
[10] S. Naduvath and K. A. Germina, "A study on topological integer additive set-labelling of graphs", Electronic journal of graph theory and applications, vol. 3, no. 1, pp. 70-84, 2015, doi: 10.5614/ ejgta.2015.3.1.8

[11] S. Naduvath, K. A. Germina, and J. Kok, "Sumset valuations of graphs and their applications", in Advanced applications of graph theory in modern society, M. Pal, S. Samanta, and A. Pal, Eds. Hershey, PA: IGI Global, 2020, pp. 208-250, doi: 10.4018/978-1-5225-9380-5.ch009

[12] M .B. Nathanson, Additive number theory: Inverse problems and the geometry of sumsets. New York, NY: Springer, 1996.

[13] A. Rosa, "On certain valuations of the vertices of a graph", in Theory of graphs, international symposium, Rome, July 1966, 1967, pp. 349-355. [On line]. Available: https:/ / bit.ly/ 3kldrJP

[14] N. K. Sudev and K. A. Germina, "On integer additive set-indexers of graphs", International journal of mathematical sciences and engineering applications, vol. 28, no. 2, pp. 11-22, 2014. [On line]. Available: https:/ / bit.ly/ 3pYc9W1

[15] N. K. Sudev and K. A. Germina, "Some new results on strong integer additive set-indexers of graphs", Discrete mathematics, algorithms and applications, vol. 7, no. 1, Art ID:1450065, 2015, doi: 10.1142/ S1 793830914500657

[16] D. B. West, Introduction to graph theory. Upper Saddle River, NJ: Prentice Hall 1996. 